\title{
Geometries and energetics of methanol-ethanol clusters: a VUV laser/time-of-flight mass spectrometry and density functional theory study ${ }^{1}$
}

\author{
Y. Liu, S. Consta, F. Ogeer, Y.J. Shi, and R.H. Lipson
}

\begin{abstract}
Hydrogen-bonded clusters, formed above liquid methanol (Me) and ethanol (Et) mixtures of various compositions, were entrained in a supersonic jet and probed using $118 \mathrm{~nm}$ vacuum ultraviolet (VUV) laser single-photon ionization/time-of-flight mass spectrometry. The spectra are dominated by protonated cluster ions, formed by ionizing hydrogen-bonded $\mathrm{Me}_{m} \mathrm{Et}_{n}$ neutrals, $m=0-4, n=0-3$, and $m+n=2-5$. The structures and energetics of the neutral and ionic species were investigated using both the all-atom optimized potential for liquid state, OPLS-AA, and the density functional (DFT) calculations. The energetic factors affecting the observed cluster distributions were examined. Calculations indicate that the large change in binding energy going from trimer to tetramer can be attributed more to pair-wise interactions than to cooperativity effects.
\end{abstract}

Key words: alcohol clusters, cluster formation, DFT calculations, mass spectrometry, vacuum ultraviolet laser.

Résumé : Les agrégats à liaisons hydrogènes qui se forment au-dessus de mélanges de méthanol (Me) et d'éthanol (Et) liquides de compositions diverses ont été entraînés dans un jet supersonique et on les a étudiés grâce à une combinaison de spectrométrie de masse à temps de vol et d'ionisation à simple photon produite par un laser ultraviolet sous vide (UVV), à $118 \mathrm{~nm}$. Les spectres sont dominés par les ions protonés, formés par l'ionisation des espèces neutres qui s'ionisent, $\mathrm{Me}_{m} \mathrm{Et}_{n}$ dans lesquelles $m=0-4, n=0-3$ et $m+n=2-5$. On a étudié les structures et les énergies tant des espèces neutres qu'ioniques à l'aide d'une combinaison calculs de la théorie de densité fonctionnelle (TDF) et du potentiel optimisé de l'état liquide pour tous les atomes (POEL-TA). On a examiné les facteurs énergétiques qui affectent les distributions d'agrégats qui ont été observés. Les calculs indiquent que le grand changement d'énergie de liaison qui est observé en passant du trimère au tétramère peut être attribué plutôt à des interactions par paire qu'à des effets de coopération.

Mots-clés : agrégats d'alcools, formation d'agrégats, calculs selon la théorie de la densité fonctionnelle (TDF), spectrométrie de masse, laser UV sous vide.

[Traduit par la Rédaction]

\section{Introduction}

Alcohol clusters have attracted considerable experimental and theoretical attention (1-3), since they often serve as prototypical molecules to study molecular association through hydrogen bonding (H-bonding). Compared to water molecules that can form as many as four H-bonds, the alkyl group in an alcohol molecule limits the number of intermolecular H-bonds each hydroxyl group can form. The

Received 13 June 2007. Accepted 15 August 2007. Published on the NRC Research Press Web site at canjchem.nrc.ca on 13 September 2007.

Y. Liu. Materials and Process Simulation Center (MSC), California Institute of Technology, 1200 East California Blvd., Pasadena, CA 91125, USA.

S. Consta, F. Ogeer, and R.H. Lipson. ${ }^{2}$ Department of Chemistry, The University of Western Ontario, London, ON N6A 5B7, Canada.

Y.J. Shi. Department of Chemistry, University of Calgary, Calgary, AB T2N 1N4, Canada.

${ }^{1}$ This article is part of a Special Issue dedicated to Professor G. Michael Bancroft.

${ }^{2}$ Corresponding author (e-mail: rlipson@uwo.ca). interplay between the formation of $\mathrm{H}$-bonding and the steric repulsion between alkyl groups determine the structure and stability of alcohol clusters. For small alcohol clusters such as methanol (Me) or ethanol (Et), H-bonds play a key role in determining the most-stable structures (4), whereas the alkyl chains have a more pronounced effect in larger clusters, as shown in our earlier studies of butanols (5). Despite the extensive amount of research that has been done on neat alcohols (1-3), the number of studies on alcohol mixtures, specifically on their vapor cluster compositions, is quite limited. In this work, VUV laser/time-of-flight (TOF) mass spectrometry was used to probe Me-Et clusters formed in the vapor phase above their liquid mixtures. Their structures and energetics were investigated using both the all-atom optimized potential for liquid state, OPLS-AA (3), and the density functional (DFT) calculations.

\section{Experimental}

The experimental arrangement used to record VUV laser mass spectra has been described in detail elsewhere (6). VUV laser mass spectrometry has been shown to be able to yield strong parent-ion signals for a wide variety of organic 
Fig. 1. A plot of the total vapor pressure of methanol-ethanol mixtures as a function of the mole fraction of ethanol $\left(X_{\mathrm{Et}}\right)$. The insert shows the deviation between the experimentally determined vapor pressures and those calculated using Raoult's Law.

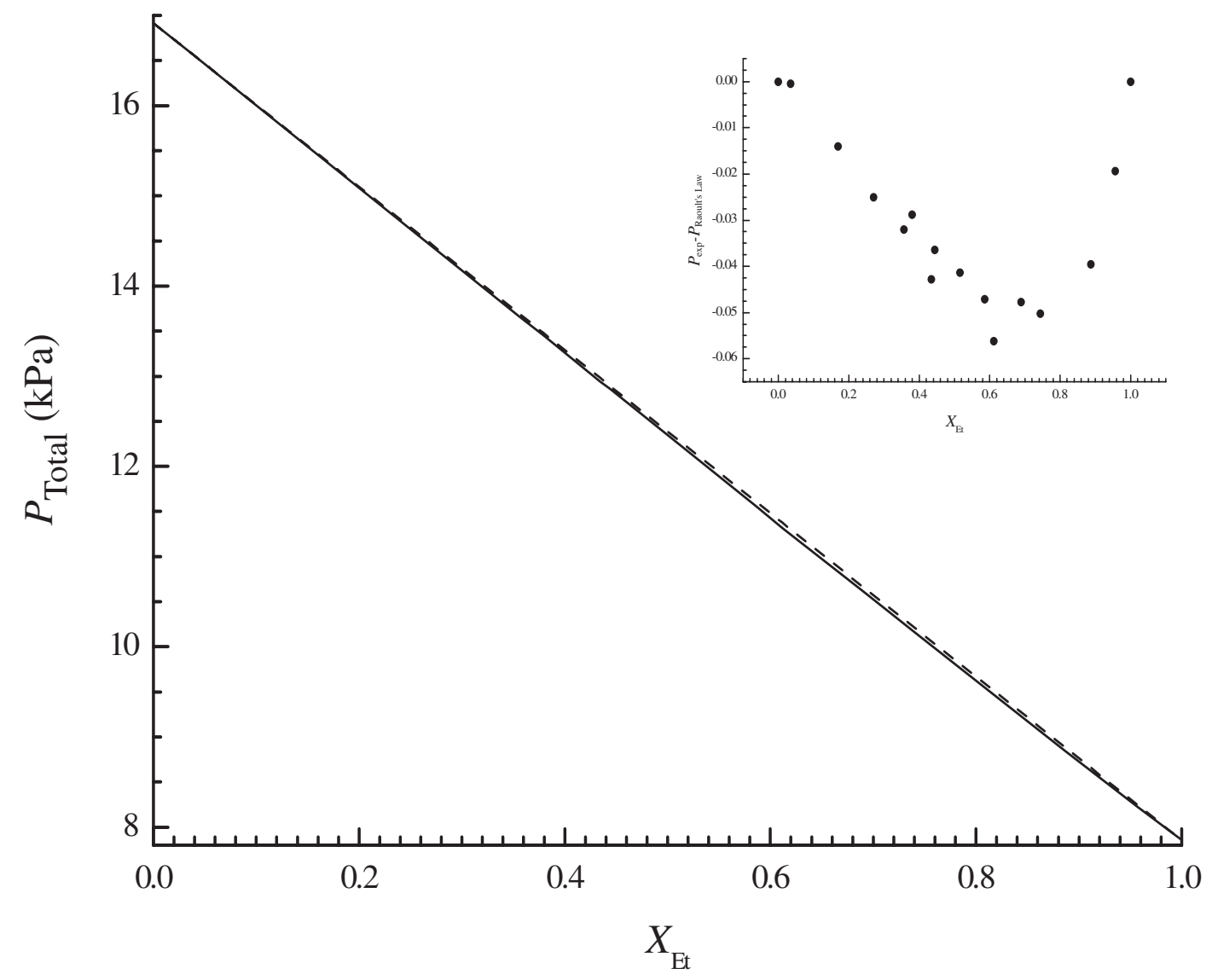

molecules having different functional groups (7). In this work, $\sim 10 \mathrm{~mL}$ of Me:Et liquids with different volume ratios ranging from 10\%:90\% to $90 \%: 10 \%$ was placed in a stainless steel vial. After the vapor-liquid equilibrium was achieved, $\sim 1 \mathrm{~atm}(1 \mathrm{~atm}=101.325 \mathrm{kPa})$ of He carrier gas was passed through the liquid mixtures. The alcohol vapor entrained in the carrier gas was subsequently expanded as a supersonic jet into the ionization region of the mass spectrometer. As shown in Fig. 1, experimentally measured Me:Et vapor pressures as a function of the mole fraction of Et $(8), X_{\mathrm{Et}}$, are very close to those predicted by Raoult's Law. The negative deviations from Raoult's law (the insert in Fig. 1), while expected for alcohol systems capable of hydrogen-bonding, are at most only $\sim 0.5 \%$ of the total vapor pressure at $X_{\mathrm{Et}} \approx 0.6$. Thus, it was felt that Raoult's Law could be reliably used to predict the vapor-phase compositions.

The jet was ionized by $118.2 \mathrm{~nm}$ VUV light generated by non-resonant third harmonic generation in a phase-matched $\mathrm{Xe} / \mathrm{Ar}$ gas cell. The positively charged ions were extracted and dispersed in a linear time-of-flight mass spectrometer $(m / \Delta m \approx 500)$. The ions were detected at the end of the $1 \mathrm{~m}$ flight tube by a microchannel plate $(\mathrm{MCP})$ to produce a mass spectrum of $\mathrm{m} / \mathrm{z}$ vs. ion intensity.

\section{Experimental results}

Typical TOF mass spectra of the Me:Et mixtures are shown in Fig. 2. Many more peaks are found in the region $\mathrm{m} / \mathrm{z}>46$ (Et monomer) than in the spectra obtained using pure Me or pure Et (4). Peak assignments are listed in Table 1 . The main observation is the presence of heterogeneous cluster ions formed from $\mathrm{Me}$ and $\mathrm{Et}$, which can be represented by a general formula $\mathrm{HMeEt}_{n}{ }^{+}$or $(m, n)$ where $m, n=1-4$, and $m+n \leq 5$. The homogenous Me and Et cluster ions are also present, and fit the general formula when either $m$ or $n$ is set to 0 . The fragment ion at $m / z=77$ is attributed to methyl loss from ethanol dimer to produce $\left[\mathrm{CH}_{2}=\mathrm{OH}^{+} \ldots \mathrm{HOC}_{2} \mathrm{H}_{5}\right]^{+}$. Most of the peaks have shoulders to slightly higher $\mathrm{m} / \mathrm{z}$, which are attributed to metastable decomposition leading to daughter fragment-ion formation inside the drift tube of the mass spectrometer.

The most-striking feature for all the TOF mass spectra of the Me:Et mixtures studied in this work is that the strongest features belong to the trimer ions of the protonated cluster. This agrees with the results obtained using neat alcohol vapors (4). The strongest peak centered at $\mathrm{m} / z=139$ in the sample with $10 \%$ Me can be assigned to $\mathrm{Et}_{3} \mathrm{H}^{+}$clusters. In the sample with $50 \% \mathrm{Me}$, the dominant features at $\mathrm{m} / \mathrm{z}=97$ and 111 , correspond to $\mathrm{Me}_{3} \mathrm{H}^{+}$and $\mathrm{Me}_{2} \mathrm{EtH}^{+}$clusters, respectively. In the sample with $90 \% \mathrm{Me}$, the mass spectrum is dominated by the peak at $m / z=97$, corresponding to $\mathrm{Me}_{3} \mathrm{H}^{+}$. It is interesting to note that the TOF mass spectrum of the 90\% Et: $10 \%$ Me binary mixture exhibits more heterogeneous cluster ions than that of the $10 \%$ Et: $90 \%$ Me mixture, which suggests that Me has a higher tendency to selfassociate in the gas-phase than Et. 
Fig. 2. VUV laser mass spectra of methanol (Me) - ethanol (Et) vapor mixtures found in equilibrium with Me-Et liquid mixtures having volume ratios of $(a)$ 10:90, (b) 50:50, and (c) 90:10. Raoult Law predicts the gas-phase molar percentage ratios to be approximately (a) 19:81, (b) 70:30, and (c) 95:5. The numbers in brackets are $(m, n)$ values corresponding to the most abundant $\mathrm{Me}_{m} \mathrm{Et}_{n} \mathrm{H}^{+}$as described in the text.
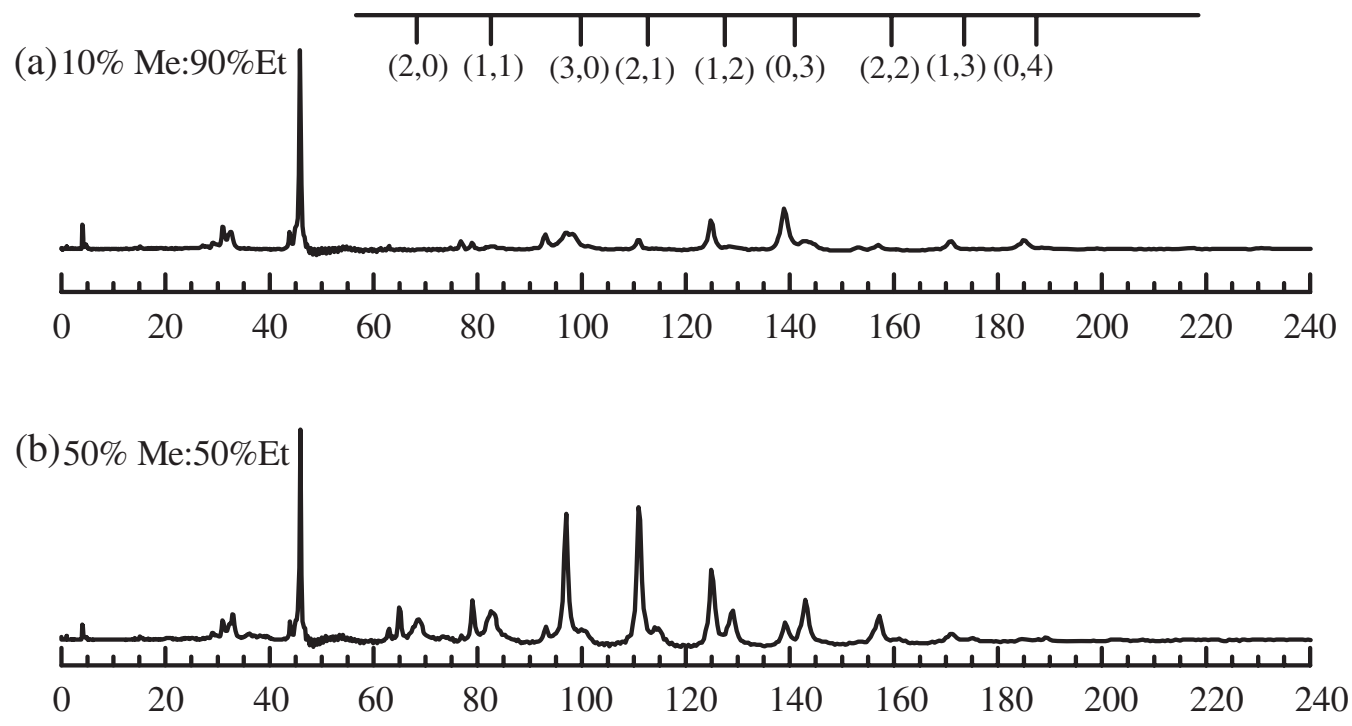

c) $90 \% \mathrm{Me}: 10 \% \mathrm{Et}$

Previous studies have shown that VUV ionization of neat Me and Et clusters yields a protonated species by the following reaction (9):

$$
\mathrm{R}_{n} \stackrel{\text { hv }}{\longrightarrow} \mathrm{R}_{n-1} \mathrm{H}^{+}+\mathrm{RO}^{-} \text {or } \mathrm{RO}^{\cdot}+\mathrm{e}^{-}
$$

where $\mathrm{R}$ denotes the alkyl chain. Similar reactions are assumed to hold for the mixed cluster as well:

$$
\begin{aligned}
& \mathrm{Me}_{m} \mathrm{Et}_{n} \stackrel{\mathrm{hv}}{\longrightarrow} \mathrm{Me}_{m-1} \mathrm{Et}_{n} \mathrm{H}^{+}+\mathrm{MeO}^{\cdot}+\mathrm{e}^{-} \\
& \mathrm{Me}_{m} \mathrm{Et}_{n} \stackrel{\mathrm{hv}}{\longrightarrow} \mathrm{Me}_{m-1} \mathrm{Et}_{n} \mathrm{H}^{+}+\mathrm{EtO}^{\cdot}+\mathrm{e}^{-}
\end{aligned}
$$

Equations [1] and [2] suggest that the probability of protonated $(N-1)$-mer formation can be correlated to the abundance of the neutral $N$-mer. Such a correlation would be invalid for van der Waals clusters because, upon ionization, they lose monomer units uncontrollably by evaporation (10). Neutral alcohol clusters, however, differ in that hydrogenbonding is $\geq 10$ times stronger than van der Waals interactions. An ionized alcohol cluster can be viewed as a solvated proton surrounded by monomer units (11). Thus, the combination of evaporative cooling due to the loss of one ligand (eqs. [1] and [2]) and solvated proton-ligand polarization makes further ligand loss difficult. Although this effect will become less important with increasing neutral-cluster size, the ion intensities recorded by VUV laser/mass spectrometry should qualitatively correlate with the neutral-cluster distri- bution in the supersonic expansion. Moreover, our work on neat methanol (4), ethanol (4), and butanol (5) vapors showed that the cluster distributions are relatively unaffected by changes in the stagnation pressure of the carrier gas used to form the supersonic expansion. This observation is supported by the work of Jackson and co-workers (12) who obtained essentially identical neat-alcohol mass spectra to ours but using very different stagnation pressures. Together, the experimental results suggest that neutral tetramers are the most-abundant clusters prior to ionization.

These neat-alcohol cluster distributions were simulated previously using a Grand Canonical Monte Carlo scheme (4, $5)$, which established that the dominance of the tetramer could only be understood if the vapor introduced by this experimental method was supersaturated. While the exact level of supersaturation has not been established quantitatively in this work, the design of experimental arrangement used to deliver the gas into the system made such a scenario a distinct possibility.

\section{Theoretical calculations}

Density functional (DFT) calculations were carried out using GAUSSIAN-98 program (13). The hybrid density functional B3LYP (14) was used, since it has been shown to provide reliable results for hydrogen-bonded systems, including neat-alcohol clusters $(15,16)$. Since an accurate de- 
Table 1. Cluster ion $\mathrm{m} / \mathrm{z}$ assignments observed in $118 \mathrm{~nm}$ VUV laser/TOF mass spectra.

\begin{tabular}{rll}
\hline & Assignment & \\
\cline { 2 - 3 } $\mathrm{HMe}_{m} \mathrm{Et}_{n}{ }^{+}$ & $(m, n)$ \\
\hline 33 & $\mathrm{HMe}^{+}$ & $(1,0)$ \\
47 & $\mathrm{HEt}^{+}$ & $(0,1)$ \\
65 & $\mathrm{HMe}_{2}{ }^{+}$ & $(2,0)$ \\
79 & $\mathrm{HMeEt}^{+}$ & $(1,1)$ \\
93 & $\mathrm{HEt}_{2}{ }^{+}$ & $(0,2)$ \\
97 & $\mathrm{HMe}_{3}{ }^{+}$ & $(3,0)$ \\
111 & $\mathrm{HMe}_{2} \mathrm{Et}^{+}$ & $(2,1)$ \\
125 & $\mathrm{HMeEt}_{2}{ }^{+}$ & $(1,2)$ \\
129 & $\mathrm{HMe}_{4}{ }^{+}$ & $(4,0)$ \\
139 & $\mathrm{HEt}_{3}{ }^{+}$ & $(0,3)$ \\
143 & $\mathrm{HMe}_{3} \mathrm{Et}^{+}$ & $(3,1)$ \\
157 & $\mathrm{HMe}_{2} \mathrm{Et}_{2}{ }^{+}$ & $(2,2)$ \\
161 & $\mathrm{HMe}_{5}{ }^{+}$ & $(5,0)$ \\
171 & $\mathrm{HMeEt}_{3}{ }^{+}$ & $(1,3)$ \\
175 & $\mathrm{HMe}_{4} \mathrm{Et}^{+}$ & $(4,1)$ \\
185 & $\mathrm{HEt}_{4}{ }^{+}$ & $(0,4)$ \\
189 & $\mathrm{HMe}_{3} \mathrm{Et}_{2}{ }^{+}$ & $(3,2)$ \\
203 & $\mathrm{HMe}_{2} \mathrm{Et}_{3}{ }^{+}$ & $(2,3)$ \\
217 & $\mathrm{HMeEt}_{4}{ }^{+}$ & $(1,4)$ \\
231 & $\mathrm{HEt}_{5}{ }^{+}$ & $(0,5)$ \\
\hline & &
\end{tabular}

scription of H-bonds requires a highly flexible basis set, the 6-311++G(d, p) basis set was used for geometry optimization, followed by single-point energy (SPE) calculations using a larger basis set, 6-311++G(3df, 2p). The energies were corrected for zero-point energy (ZPE) and basis-set superposition error (BSSE) (17). Geometry optimizations were also done using the OPLS-AA force field. Comparison with the DFT results can provide an evaluation of the empirical potentials used in the OPLS-AA force field.

\section{(a) Structures of $\mathrm{Me}_{m} \mathrm{Et}_{n}(m=0-4, n=0-4, m+n=$} 2-5) isomers

Alcohol clusters exhibit structural isomerism. For example, the ethanol monomer has a trans $(\mathrm{t})$ and a gauche $(\mathrm{g})$ conformation in which the trans isomer is slightly more stable than the gauche. Global minima on the potential energy surface (PES) for the structural isomers were found by first performing Molecular Dynamics (MD) simulations in a NVT ensemble at $T=100 \mathrm{~K}$ using the OPLS-AA force field. Several tens of local minima on the PES were then selected as the initial conformations for geometry optimizations using the OPLS-AA force field. The structures obtained were further optimized by DFT/B3LYP calculations. The optimized structures among the most-stable isomers are shown in Figs. $3 a-3 d$. Except for the dimers, which form open structures, the most-stable structures for the trimers through to the pentamers are monocyclic rings in which each molecule has two H-bonds. The oxygen atoms in these structures act as both a proton donor and an acceptor. The number of $\mathrm{H}$-bonds is a maximum in the cyclic trimer, which agrees reasonably with intuition. However, cyclic tetramers and pentamers in fact do not have the maximum number of $\mathrm{H}$ bonds possible. For example, the monocyclic pentamer is more stable than the branched cyclic species even though one molecule in the branched cyclic structure has three $\mathrm{H}$ bonds. It should be appreciated that another common feature of the monocyclic structures is that the alkyl groups are arranged to minimize steric hindrance. A similar behavior is found for water clusters (18).

Figures $4 a-4 d$ show the dependences of the nearestneighbour $\mathrm{O}-\mathrm{O}$ separations, the $\mathrm{H}$-bonded $\mathrm{O}-\mathrm{H}$ bond lengths, the covalent $\mathrm{O}-\mathrm{H}$ bond lengths, and the $\mathrm{OHO}$ angles on cluster size. These values were calculated by taking averages over various mixture clusters. As the cluster size increases, the $\mathrm{O}-\mathrm{O}$ distance $\left(r_{\mathrm{O}-\mathrm{O}}\right)$ and the $\mathrm{H}$-bond length $\left(r_{\mathrm{O}-\mathrm{H}}\right)$ decrease, while the covalent $\mathrm{O}-\mathrm{H}$ bond lengths $\left(r_{\mathrm{O}-\mathrm{H}}^{\prime}\right)$ increase slightly. These trends indicate that the monocyclic structures contract because of H-bond shortening as the cluster becomes larger. This structure change implies that $\mathrm{H}$ bonding becomes stronger with the increasing cluster size, which is consistent with the trends of the average binding energies per molecule (see later). On the other hand, the $\mathrm{O}-$ $\mathrm{O}$ repulsions do not favor the contractions energetically. The interplay between $\mathrm{H}$-bonding and $\mathrm{O}-\mathrm{O}$ repulsion results in the tetramers and the pentamers having comparable $\left\langle E_{\mathrm{b}}\right\rangle$ and $\left\langle r_{\mathrm{O}-\mathrm{O}}\right\rangle$. A similar trend was also found in our previous studies of the $n$-butanol and iso-butanol isomers, but not for sec-butanol and tert-butanol (5). Since the sec-butanol and tert-butanol have short and bulky alkyl chains, steric repulsion makes the $\mathrm{O}-\mathrm{O}$ separations larger in the pentamers than in the tetramers. The structural changes in small alcohol clusters resemble those of water including a systematic contraction of the nearest-neighbour $\mathrm{O}-\mathrm{O}$ separation with increasing cluster size, and a red shift of the $\mathrm{H}$-bonded $\mathrm{OH}$ stretch frequency $(19,20)$.

The proton positions were located in this work by measuring the average $\mathrm{OHO}$ angle (Fig. $4 d$ ). In the dimers, the protons lay approximately along the $\mathrm{O}-\mathrm{O}$ axes to form linear H-bonds. The trimer protons are so close in this cluster that repulsion forces them outward to off-axis positions. The deviations from the $\mathrm{O}-\mathrm{O}$ axes reduce quickly however with the increasing cluster size, which is indicated by an increasing $\mathrm{OHO}$ angle going from the trimer to the pentamer. In the pentamer, the OHO angles are essentially restored to the values found for the dimer. This implies that in the pentamer, the proton-proton separations are sufficiently large that the repulsion between them has become negligible. Extrapolation of these trends suggests that even larger monocyclic structures should have very similar bond distances and average binding energies per molecule. Of course, it may be that these larger clusters would rather adopt more compact shapes, for example, bicyclic or cage-like structures.

Lastly, the cluster structures obtained from the OPLS-AA and the DFT calculations were compared. The OPLS-AA model underestimates cluster covalent $\mathrm{O}-\mathrm{H}$ bond lengths by at least 0.01 although the trend is similar to that calculated by DFT (Fig. 4c). The OPLS-AA also underestimates the $\mathrm{H}$ bond length and the nearest-neighbour $\mathrm{O}-\mathrm{O}$ distance for the dimer. These deviations correlate with the overestimated OPLS-AA binding energies. Yet, except for the deviations mentioned earlier, the OPLS-AA model predicts the microscopic cluster structures almost as well as DFT. 
Fig. 3. Optimized structures of $\mathrm{Me}_{m} \mathrm{Et}_{n}(m=0-4, n=0-3, m+n=2-5)$ clusters found by DFT/B3LYP calculations: (a) dimers, (b) trimers, $(c)$ tetramers, and $(d)$ pentamers. The unit of bond length is $\AA$, and the angles are in degrees.

(a)

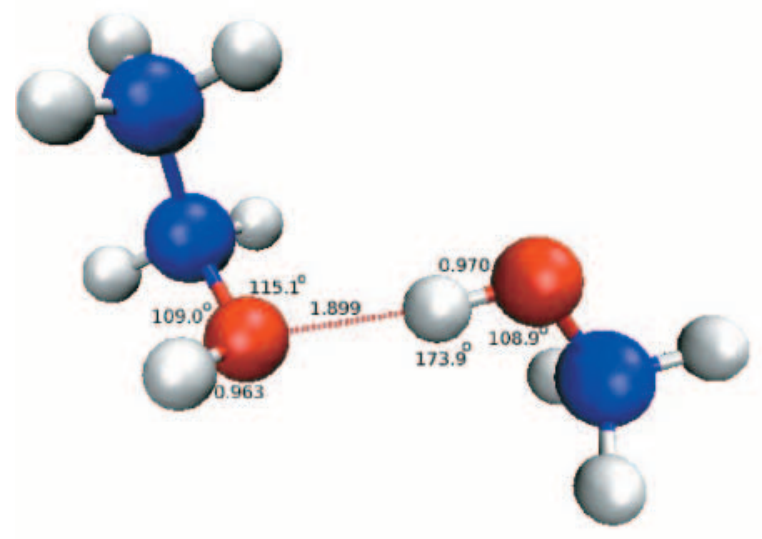

(c)

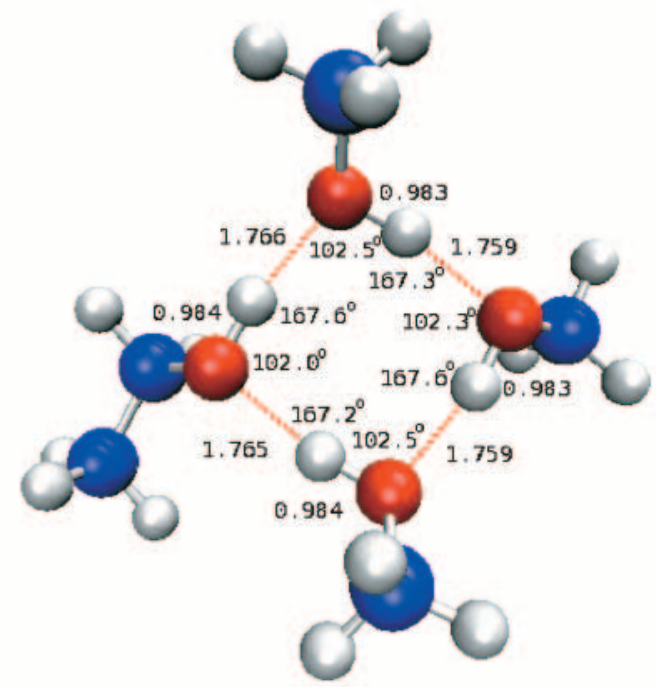

(b) Structures of protonated clusters

As noted earlier, the protonated trimer ions are the most dominant cluster ions detected by VUV laser mass spectrometry. The optimized structures for $\mathrm{Me}_{3} \mathrm{H}^{+}, \mathrm{Et}_{3} \mathrm{H}^{+}$, and $\mathrm{Me}_{2} \mathrm{EtH}^{+}$, respectively, are shown in Figs. $5 a-5 d$. Unlike the neutral clusters, the protonated trimers form open structures that maximize the alkyl group separations and minimize proton-proton repulsions. The H-bonds are noticeably shorter than those in the neutral clusters, and the covalent $\mathrm{O}-\mathrm{H}$ bonds are slightly stretched. As a result, the nearestneighbour $\mathrm{O}-\mathrm{O}$ distances are also contracted in the protonated clusters relative to their neutral counterparts.

Energetics of $\mathrm{Me}_{m} \mathrm{Et}_{n}(m=0-4, n=0-4, m+n=2-5)$ isomers

The binding energy of a cluster, $E_{\mathrm{b}}^{\prime}$, is defined as the difference between the total energy of the cluster and those of its isolated components: (b)

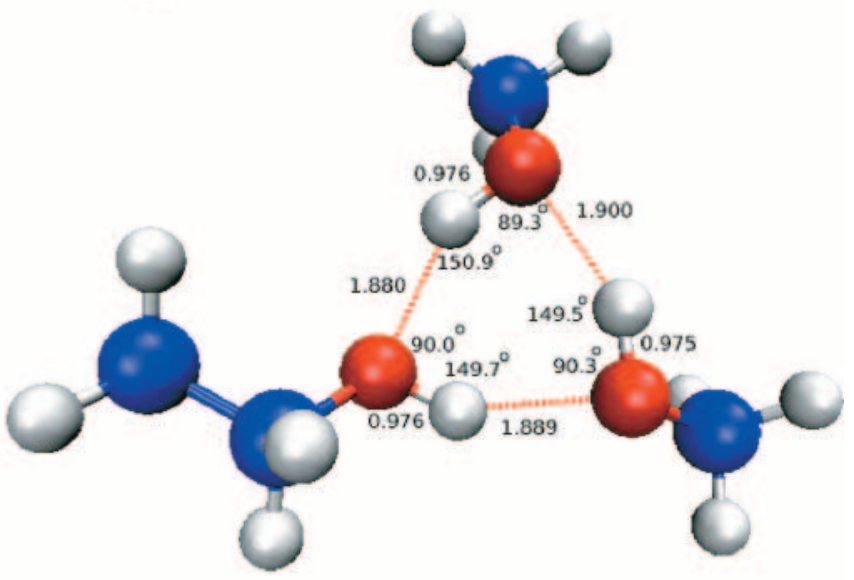

(d)

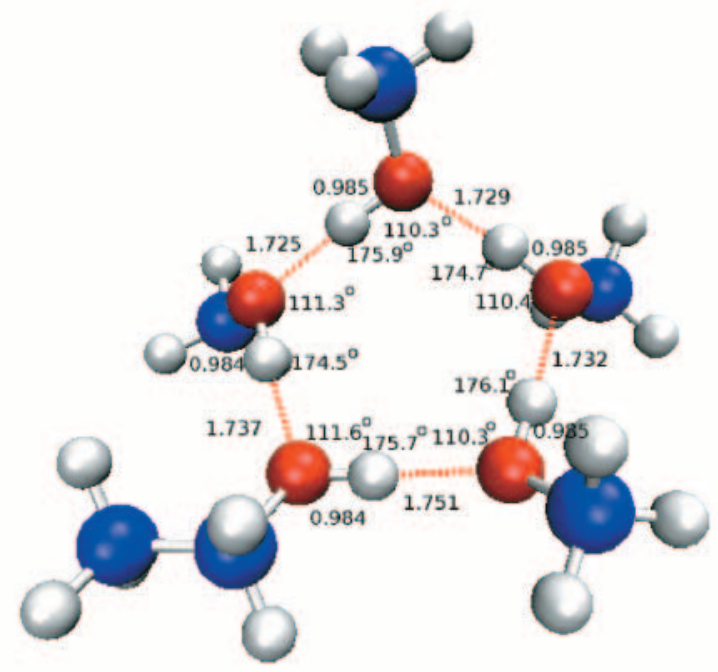

[3]

$$
\begin{aligned}
E_{\mathrm{b}}^{\prime}\left[\mathrm{Me}_{m} \mathrm{Et}_{n}\right]=E_{\mathrm{tot}}\left[\mathrm{Me}_{m} \mathrm{Et}_{n}\right]-m E_{\mathrm{tot}}[\mathrm{Me}] & \\
& -n E_{\mathrm{tot}}[\mathrm{Et}]
\end{aligned}
$$

where $E_{\text {tot }}\left[\mathrm{Me}_{m} \mathrm{Et}_{n}\right], E_{\mathrm{tot}}[\mathrm{Me}]$, and $E_{\mathrm{tot}}[\mathrm{Et}]$ are the total energies of the mixed cluster, methanol monomer, and ethanol monomer, respectively. The binding energy per molecule can be calculated from $E_{\mathrm{b}}\left[N_{(m+n)}\right]=E_{\mathrm{b}}^{\prime}\left[\mathrm{Me}_{m} \mathrm{Et}_{n}\right] /(m+n)$. For monocyclic clusters that have the same number of molecules and H-bonds (other than dimer), $E_{\mathrm{b}}\left[N_{(m+n)}\right]$ represents the strength of a H-bond. The incremental binding energy is then defined as follows:

$$
E_{\mathrm{ib}}\left[N_{(m+n)}\right]=E_{\mathrm{b}}\left[N_{(m+n)}\right]-E_{\mathrm{b}}\left[N_{(m+n-1)}\right]
$$

and is a measure of the change in the H-bond strength because of the addition of a monomer to the cluster.

The binding energy can be further understood in terms of many-body interactions. The binding energy of a $\mathrm{Me}_{m} \mathrm{Et}_{n}$ binary cluster can be decomposed as follows (18): 
Fig. 4. (a) Average $\mathrm{O}-\mathrm{O}$ distances, $(b)$ average H-bond lengths, $(c)$ average covalent $\mathrm{O}-\mathrm{H}$ bond lengths, and $(d)$ average $\mathrm{OHO}$ angles, as a function of cluster size calculated using the OPLS-AA model and by DFT.
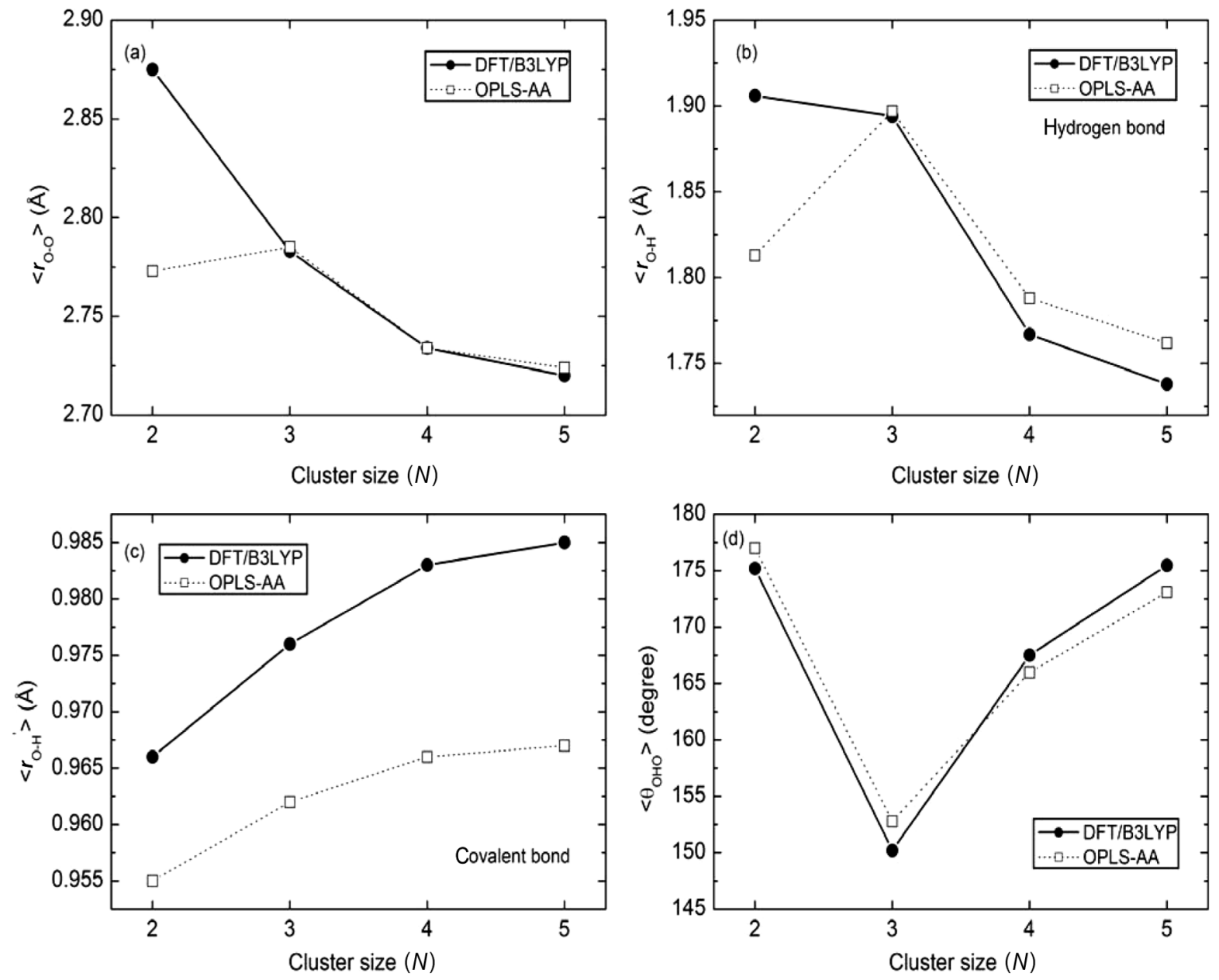

[5]

$$
\begin{aligned}
& E_{\mathrm{b}}^{\prime}\left[\mathrm{Me}_{m} \mathrm{Et}_{n}\right] \equiv \sum_{i=1}^{m+n} E(i)-m E_{\mathrm{tot}}(\mathrm{Me})-n E_{\mathrm{tot}}(\mathrm{Et}) \\
& +\sum_{i=1}^{m+n-1} \sum_{j>1}^{m+n} \Delta^{2} E(\mathrm{ij})+\sum_{i=1}^{m+n-2} \sum_{j>1}^{m+n} \sum_{k>j}^{m+n} \Delta^{3} E(\mathrm{ijk}) \\
& +\sum_{i=1}^{m+n} \sum_{j>l}^{m+n} \sum_{k>j}^{m+n} \sum_{l>k}^{m+n} \Delta^{4} E(\mathrm{ijkl})+\cdots+\Delta^{m+n} E(m, n)
\end{aligned}
$$

$$
\begin{aligned}
& -\left[\Delta^{2} E(\mathrm{ij})+\Delta^{2} E(\mathrm{ik})+\Delta^{2} E(\mathrm{il})\right] \\
& -\left[\Delta^{2} E(\mathrm{jk})+\Delta^{2} E(\mathrm{jl})+\Delta^{2} E(\mathrm{kl})\right] \\
& -\left[\Delta^{3} E(\mathrm{ijk})+\Delta^{3} E(\mathrm{ijl})+\Delta^{3} E(\mathrm{ikl})+\Delta^{3} E(\mathrm{jkl})\right]
\end{aligned}
$$

The cooperativity is defined as the sum of all non-additive terms with orders higher than the two-body term. The resultant binding energies were computed for $\mathrm{Me}_{m} \mathrm{Et}_{n}(m=0-4$, $n=0-4, m+n=2-5$ ) isomers, and are listed in Table 2. Excellent agreement with the experimental values for pure methanol and ethanol dimers (21) were obtained when the binding energies were calculated after geometry optimizations that included corrections for ZPEs but not BSSE. Moreover, the calculated binding energy of methanol dimer differed by only $0.05 \mathrm{kcal} / \mathrm{mol}$ from the value obtained from MP2/VTZ(2df, 2p) and MP2/ESPB calculations (15). In general, the binding energies among various isomers are very similar, with energy variations typically $<0.3 \mathrm{kcal} / \mathrm{mol}$. The gauche mixed dimer was found to be slightly more stable than the trans species, while the opposite trend was observed for the mixed trimers.

The relative stability of the different clusters was determined by the energy calculations done using both the OPLSAA and the DFT/B3LYP methods. Plots of the average binding energy/molecule, and the average incremental binding 
Fig. 5. Optimized structures of the protonated clusters obtained by DFT/B3LYP calculations: (a) $\mathrm{Me}_{3} \mathrm{H}^{+},\left(\right.$b) $\mathrm{Et}_{3} \mathrm{H}^{+}$, (c) $\mathrm{Me}_{2} \mathrm{EtH}^{+}$, and (d) $\mathrm{Me}_{2} \mathrm{H}^{+}$Et. The unit of bond length is $\AA$, and angles are given in degrees.

(a)

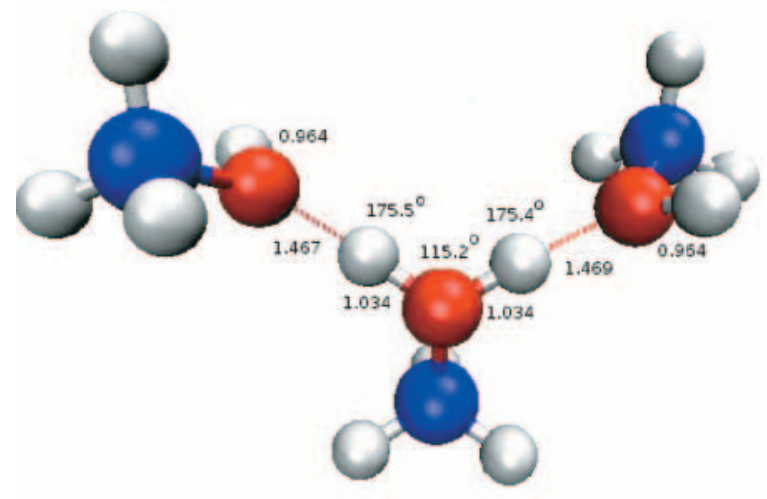

(c)

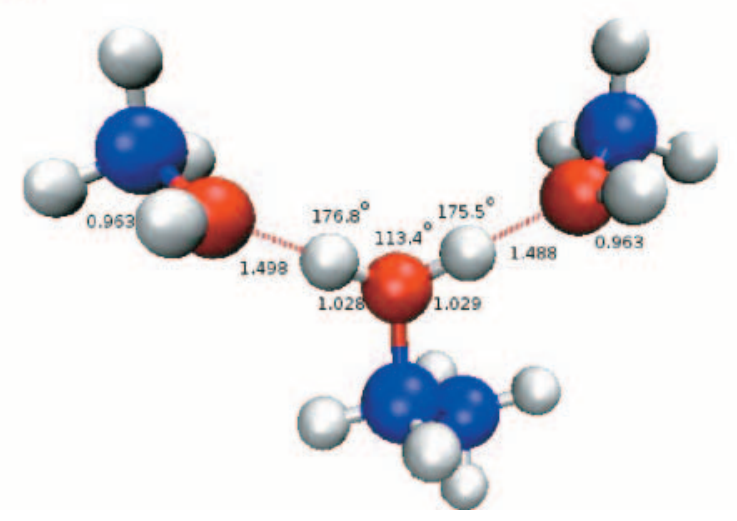

energy vs. cluster size are shown in Figs. $6 a$ and $6 b$, respectively. The energies were averaged per cluster over the different isomers. The average incremental binding energy clearly maximizes at $N_{(m+n)}=4$. Therefore, the large energy gain due to the formation of the tetramers should be associated with its abundance in the vapor, along with the supersaturation effect discussed before. A similar energy trend has been reported for neat methanol and water vapors $(1,2)$. It should be noted that methanol prefers to form larger clusters than water (1). This difference implies that entropy as well as energetics may also play a role in determining cluster-size distributions in alcohols.

The results obtained using the empirical force field were compared with those obtained by DFT. As shown in Fig. $6 b$, both the OPLS-AA and DFT calculations predict a similar trend in the size dependence of the incremental binding energy. Strikingly, the incremental binding energies for the tetramer obtained by the two different approaches agree remarkably well. As this value describes the relative stability of the trimers and tetramers, it is critical in understanding the exceptional stability of the tetramer. The OPLS-AA model generally overestimates the binding energy per molecule (Fig. 6a). The largest discrepancy is found for the dimer. This should translate into a large deviation of the computed dimer $\mathrm{OH}$ stretching modes from the experimental values (2). This disagreement, however, is not surprising because the OPLS-AA potential parameters were originally fit- (b)

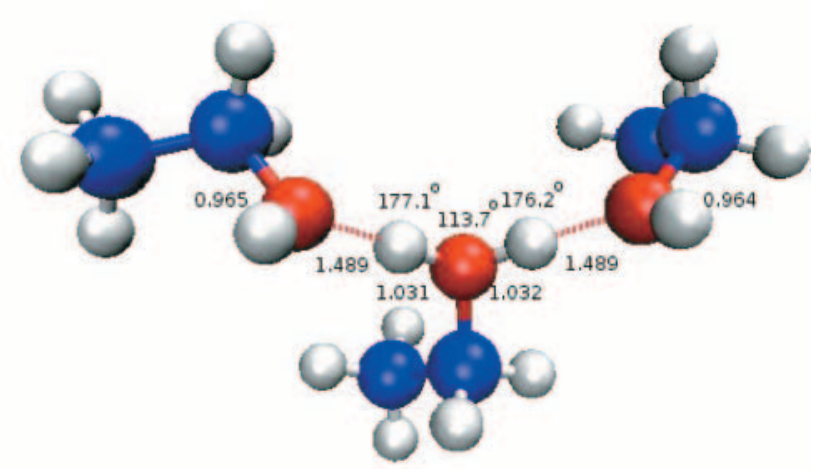

(d)

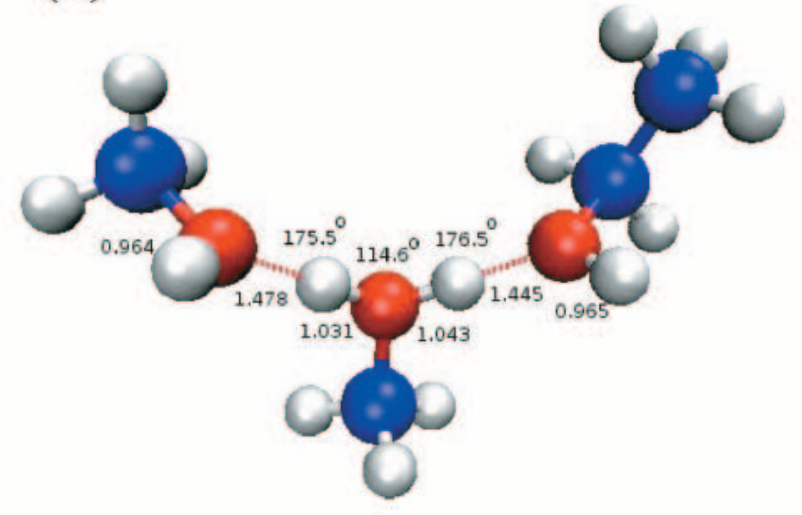

ted to reproduce the properties of liquids. The origin of this error probably comes from an overestimation of the static dipole moment in the force field parameters (2). The OPLSAA model describes the stability of large clusters better because, in the gas-phase, large clusters usually adopt cyclic structures, which closely resemble their liquid-phase counterparts. However, further comparisons with DFT show that the OPLS-AA model fails to predict the relative stabilities among the clusters of equal $N$, and the relative stability of the structural isomers associated with a given cluster composition. Here, the energy differences are generally very small, and this suggests therefore, that the OPLS-AA model is only qualitatively reliable for predicting the stability of clusters of different sizes. While the OPLS-AA model does describe the relative stability between trimers and tetramers well, significant differences between the calculated OPLS-AA and DFT energies exist. Careful tests should therefore be taken when using the OPLS-AA energies to describe the stability of the cluster.

\section{Discussion and conclusions}

VUV single-photon ionization and TOF mass spectrometry were used to detect gas-phase protonated methanol and ethanol cluster ions. Optimized structures for these neutral precursors have been calculated. Not surprisingly the moststable neutral mixed clusters are monocyclic, similar to that 
Table 2. Binding energies of $\mathrm{Me}_{m} \mathrm{Et}_{n}(m=0-4, n=0-4, m+n=2-5)$ isomers. The subscript $\mathrm{t}$ or $\mathrm{g}$ denotes an ethanol molecule with a trans or gauche conformation, respectively. The values in parentheses are the binding energies after ZPE correction. All energies are in units of $\mathrm{kcal} / \mathrm{mol}$.

\begin{tabular}{|c|c|c|c|c|}
\hline Isomer & & $E_{\mathrm{b} 1}{ }^{a}$ & $E_{\mathrm{b} 2}^{b}$ & BSSE \\
\hline$(\mathrm{MeOH})_{2}$ & $A$ & $-5.83(-4.45)$ & $-4.93(-3.55)^{c}$ & 0.24 \\
\hline \multirow[t]{3}{*}{$(\mathrm{EtOH})_{2}$} & $A_{\mathrm{tt}}$ & $-5.61(-4.31)$ & $-4.48(-3.17)^{c}$ & 0.23 \\
\hline & $B_{\text {tg }}$ & $-5.71(-4.43)$ & $-4.60(-3.33)^{c}$ & 0.24 \\
\hline & $C_{\mathrm{gg}}$ & $-5.49(-4.31)$ & $-4.41(-3.23)^{c}$ & 0.25 \\
\hline \multirow[t]{3}{*}{$(\mathrm{MeOH})(\mathrm{EtOH})$} & $A_{\mathrm{t}}$ & $-5.81(-4.40)$ & $-5.05(-3.63)$ & 0.26 \\
\hline & $B_{\mathrm{g}}$ & $-5.83(-4.42)$ & $-5.15(-3.74)$ & 0.25 \\
\hline & $C_{\mathrm{t}}$ & $-5.65(-4.35)$ & $-4.76(-3.45)$ & 0.25 \\
\hline \multirow[t]{6}{*}{$(\mathrm{MeOH})(\mathrm{EtOH})_{2}$} & $A_{\mathrm{tt}}$ & $-16.60(-13.27)$ & $-14.79(-11.47)$ & 0.78 \\
\hline & $B_{\mathrm{tt}}$ & $-16.53(-13.29)$ & $-14.73(-11.49)$ & 0.87 \\
\hline & $C_{\mathrm{tg}}$ & $-16.40(-13.12)$ & $-14.61(-11.33)$ & 0.78 \\
\hline & $D_{\mathrm{tg}}$ & $-16.35(-13.16)$ & $-14.64(-11.46)$ & 0.74 \\
\hline & $E_{\mathrm{gg}}$ & $-16.23(-13.06)$ & $-14.51(-11.34)$ & 0.72 \\
\hline & $F_{\mathrm{gg}}$ & $-16.15(-13.02)$ & $-14.34(-11.22)$ & 0.72 \\
\hline \multirow[t]{3}{*}{$(\mathrm{MeOH})_{2}(\mathrm{EtOH})$} & $A_{\mathrm{t}}$ & $-16.86(-13.47)$ & $-14.96(-11.58)$ & 0.77 \\
\hline & $B_{\mathrm{g}}$ & $-16.71(-13.33)$ & $-14.85(-11.45)$ & 0.77 \\
\hline & $C_{\mathrm{g}}$ & $-16.65(-13.27)$ & $-14.69(-11.31)$ & 0.76 \\
\hline \multirow[t]{2}{*}{$(\mathrm{MeOH})(\mathrm{EtOH})_{3}$} & $A_{\mathrm{ttt}}$ & $-29.23(-24.32)$ & $-26.27(-21.36)$ & 1.25 \\
\hline & $B_{\mathrm{ttg}}$ & $-29.15(-24.22)$ & $-26.15(-21.22)$ & 1.26 \\
\hline \multirow[t]{2}{*}{$(\mathrm{MeOH})_{2}(\mathrm{EtOH})_{2}$} & $A_{\mathrm{tt}}$ & $-29.61(-24.52)$ & $-26.54(-21.45)$ & 1.28 \\
\hline & $B_{\mathrm{tt}}$ & $-29.62(-24.49)$ & $-26.54(-21.41)$ & 1.26 \\
\hline$(\mathrm{MeOH})_{3}(\mathrm{EtOH})$ & $A_{\mathrm{t}}$ & $-29.97(-24.82)$ & $-26.76(-21.61)$ & 1.29 \\
\hline$(\mathrm{MeOH})_{2}(\mathrm{EtOH})_{3}$ & $A_{\mathrm{ttt}}$ & $-38.68(-32.37)$ & $-32.64(-26.33)$ & 1.51 \\
\hline$(\mathrm{MeOH})_{3}(\mathrm{EtOH})_{2}$ & $A_{\mathrm{tt}}$ & $-39.33(-32.91)$ & $-33.13(-26.71)$ & 1.53 \\
\hline$(\mathrm{MeOH})_{4}(\mathrm{EtOH})$ & $A_{\mathrm{t}}$ & $-39.76(-33.21)$ & $-33.41(-26.89)$ & 1.54 \\
\hline
\end{tabular}

${ }^{a}$ Binding energy calculated after geometry optimization using B3LYP/6-311++G(d, p).

${ }^{b}$ Binding energy calculated after geometry optimization using B3LYP/6-311++G(d, p) followed by a single point energy calculation using B3LYP/6-311++G(3df, 2p).

${ }^{c}$ Experimental binding energies for pure methanol and ethanol dimers (23) lie between -3.20 to $-3.50 \mathrm{kcal} / \mathrm{mol}$, and -3.39 to $-3.99 \mathrm{kcal} / \mathrm{mol}$, respectively.

found for neat-alcohol clusters (4). These structures are also consistent with recent infrared studies that suggest that the $\mathrm{OH}$ groups of the monomers involved in neat methanol and ethanol clusters larger than the dimer are all involved in hydrogen bonding (22-24). The protonated trimer ions correspond to the most abundant species detected by VUV laser/time-of-flight mass spectrometry. The correlation shown in eq. [1] between a neutral $N$-mer and a protonated $(N-1)$-mer ion suggests that the mixed alcohol tetramers are "magic" clusters. This conclusion holds for neat methanol and ethanol tetramers because these size clusters (with $S_{4}$ symmetry) are expected to exhibit a maximum hydrogenbonding cooperativity effect, and a minimum ring strain (1, 2).

The neutral mixed cluster binding energies were decomposed into the terms representing the many-body interactions (see eqs. [5-8]) to understand the size-dependence trend found here for the incremental binding energy. Table 3. shows the average binding energies and their components calculated by DFT (without ZPE corrections) for the alcohol clusters ranging from the dimer to the pentamer. It can be seen again that the difference in the binding energies for the $N$-mer and $(N-1)$-mer maximizes for the tetramer. Further examination shows that the differences in the pair-wise additive contributions have the same trend as the binding-energy differences, whereas the differences in the non-additive contributions, often termed cooperativity effects, does not exhibit this trend. This observation suggests that the large change in energy going from the trimer to the tetramer is mainly due to pair-wise interactions rather than higher-order cooperativity effects; that is, the tetramer stability is mainly a balance between the energy gained through attractive $\mathrm{H}$ bonding vs. the repulsion between the oxygen atoms within the cluster.

It is instructive to compare the relative contributions of the pair-wise additive and non-additive terms, rather than their absolute values. Table 4. lists the percentage contribution of the many-body terms to the binding energy, which were averaged over various alcohol clusters, along with the corresponding values for water clusters taken from ref. 18. It can be seen that the repulsive contribution due to the relaxation term becomes more pronounced going from the dimer to the pentamer. This indicates that for the larger clusters, the individual monomers deform considerably relative to their isolated conformations. These deformations include the stretching of the covalent $\mathrm{O}-\mathrm{H}$ bonds (Fig. 4c). The additive 
Table 3. Average binding energies and their many-body components for $\operatorname{Me}_{m} \mathrm{Et}_{n}(m=0-4, n=0-4, m+n=2-5)$ clusters (in $\mathrm{kcal} / \mathrm{mol}$ ). The binding energies $E_{\mathrm{b} 2}$ correspond to those in Table 2 (without ZPE corrections). $E_{\mathrm{r}}$ is the relaxation term defined in eq. [5]. $\left\langle{ }^{\mathrm{n}} E>\right.$ are the $n$-body terms defined in eqs. [5-8]. $E_{\mathrm{c}}$ corresponds to the non-additive terms. $\left\langle E_{\mathrm{b} 2}\right\rangle,\left\langle{ }^{2} E\right\rangle$, and $E_{\mathrm{c}}$ are the differences of average binding energies, two-body terms, and non-additive terms, respectively. The positive signs for the relaxation terms denote repulsive contributions to the energy.

\begin{tabular}{lllllllllll}
\hline$m+n$ & $\left\langle E_{\mathrm{b} 2}\right\rangle$ & $E_{\mathrm{r}}$ & $\left\langle\Delta^{2} E\right\rangle$ & $\left\langle\Delta^{3} E\right\rangle$ & $\left\langle\Delta^{4} E\right\rangle$ & $\left\langle\Delta^{5} E\right\rangle$ & $E_{c}$ & \multicolumn{1}{c}{$\left.\Delta<E_{\mathrm{b} 2}\right\rangle$} & $\left.\Delta<\Delta^{2} E\right\rangle$ & $\Delta E_{c}$ \\
\hline 2 & -4.98 & 0.14 & -5.12 & - & - & - & - & -4.98 & -5.12 & - \\
3 & -14.69 & 0.56 & -12.15 & -3.10 & - & - & -3.10 & -9.71 & -7.03 & -3.10 \\
4 & -26.45 & 1.43 & -21.53 & -3.89 & -2.46 & - & -6.53 & -11.76 & -9.38 & -3.25 \\
5 & -33.06 & 2.44 & -25.50 & -7.63 & -1.76 & -0.61 & -10.00 & -6.61 & -3.97 & -3.65 \\
\hline
\end{tabular}

Table 4. Percentage contribution of many-body terms to the binding energy of $\mathrm{Me}_{m} \mathrm{Et}_{n}(m=0-4, n=0-4, m+n=2-5)$ clusters. The notations have the same meanings as those in Table 3. The negative sign indicates that the relaxation term has a repulsive contribution. The values in parenthesis are the corresponding values for $\left(\mathrm{H}_{2} \mathrm{O}\right) n(n=2-5)$ clusters, which are taken from ref. 19.

\begin{tabular}{lllllll}
\hline$m+n$ & Theory level & $E_{\mathrm{r}}$ & $\left\langle{ }^{2} E\right\rangle$ & $\left\langle{ }^{3} E\right\rangle$ & $\left\langle{ }^{4} E\right\rangle$ & $\left\langle{ }^{5} E\right\rangle$ \\
\hline 2 & B3LYP(MP2) & $-2.8(-0.8)$ & $102.8(100.8)$ & - & - & - \\
3 & B3LYP(MP2) & $-3.8(-2.7)$ & $82.7(85.1)$ & $21.1(17.6)$ & - & - \\
4 & B3LYP(MP2) & $-5.4(-4.1)$ & $81.4(76.3)$ & $14.7(25.6)$ & $9.3(2.2)$ & - \\
5 & B3LYP(HF) & $-7.4(-1.4)$ & $77.1(78.9)$ & $23.1(20.4)$ & $5.3(2.1)$ & $1.9(0.01)$ \\
\hline
\end{tabular}

Fig. 6. (a) Average binding energy per molecule, $\left\langle E_{\mathrm{b}}\right\rangle$, and (b) average incremental binding energy, $\left\langle E_{\mathrm{ib}}\right\rangle$, as a function of cluster size obtained by DFT/B3LYP and OPLS-AA calculations.

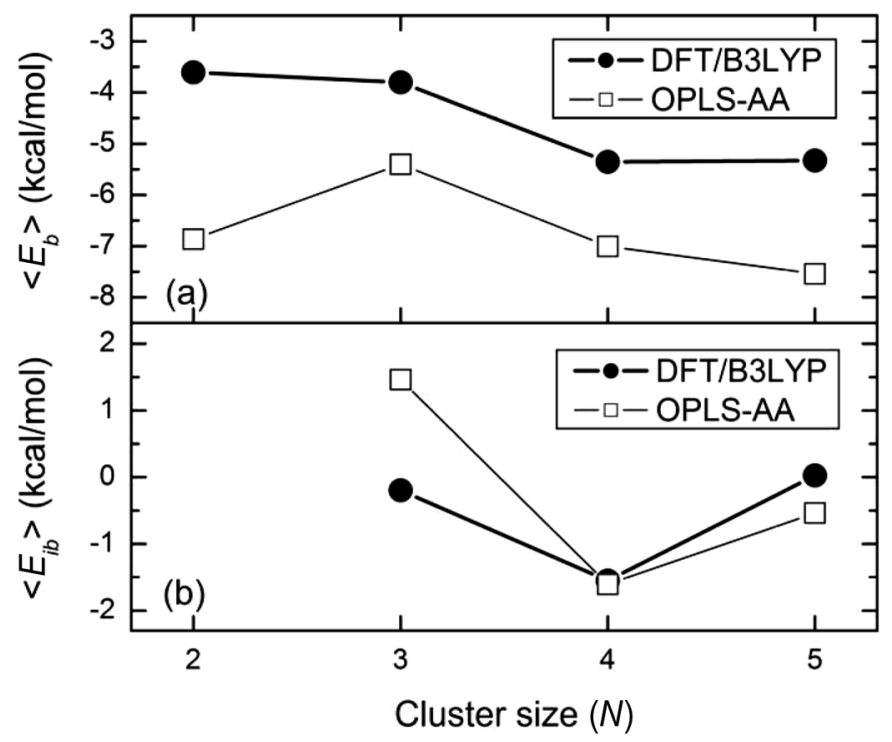

contribution to the binding energy represented by the twobody term decreases, while the non-additive contribution increases with the increasing cluster size. The non-additive interactions (cooperativity) can contribute significantly (as high as $20 \%-30 \%$ ) in both the alcohol and the water clusters. The four- and five-body terms also provide nonnegligible contributions (7\%-9\%) in the alcohol clusters, whereas these contributions are less than $3 \%$ in the water clusters. In other words, the four- and five-body terms in the alcohol clusters contribute $\sim 30 \%$ of the cooperativity effect in alcohol clusters. In the water clusters, however, the contributions from these high-order terms are less than $10 \%$. This analysis shows that cooperativity effects in both alco- hol and water clusters are significant. Nevertheless, the alcohol-cluster binding energies converge more slowly than those for water clusters. To capture the cooperativity effects in water clusters, it was deemed sufficient to consider terms up to and including three-body interactions to design a force field (18). The effect of even higher-order terms, such as the four-body interaction, must be included for alcohol clusters to obtain a similar accuracy.

It is well known that different levels of theory can have a considerable effect on the decomposition of the binding energy: second-order many-body perturbation (MP2) level that includes electron correlation generally will decrease the additive contribution relative to a Hartree-Fock (HF) calculation (18) but will increase the non-additive contribution. The DFT/B3LYP results here are considered to be comparable to those obtained by MP2.

As noted earlier, the open structure calculated for the mixed ionic trimers and shown in Fig. 5 can be viewed as a solvated proton. This behavior is also observed for the neat protonated dimers (25). The methanol protonated trimer in particular is a "magic" structure, as it can be regarded as a proton with a complete first-solvation shell $(11,26)$. Infrared spectroscopy reveals that the free and hydrogen-bonded $\mathrm{OH}$ stretching frequencies of the neat cluster ions blue-shift with increasing $N(22,24)$. The mixed protonated ions are expected to exhibit a similar spectroscopic signature.

\section{Acknowledgements}

The authors acknowledge the financial support of the Natural Sciences and Engineering Research Council of Canada (NSERC) and Shared Hierarchical Academic Research Computing Network (SHARCNET) at UWO. One of us (S.C.) is grateful for funding through a Premier's Research Excellence Award. 


\section{References}

1. L.A. Curtiss and M. Blander. Chem. Rev. 88, 827 (1988).

2. U. Buck, J.-G. Siebers, and R.J. Wheatley. J. Chem. Phys. 108, 20 (1998).

3. W.L. Jorgensen. J. Phys. Chem. 90, 1276 (1986).

4. Y.J. Shi, S. Consta, A.K. Das, B. Mallik, D. Lacey, and R.H. Lipson. J. Chem. Phys. 116, 6990 (2002), and references within.

5. G.S. Fanourgakis, Y.J. Shi, S. Consta, and R.H. Lipson. J. Chem. Phys. 119, 6597 (2003).

6. R.H. Lipson, S.S. Dimov, P. Wang, Y.J. Shi, D. M. Mao, X.K. $\mathrm{Hu}$, and J. Vanstone. Instrum. Sci. Technol. 28, 85 (2000).

7. Y.J. Shi and R.H. Lipson. Can J. Chem. 83, 1891 (2005).

8. Z.S. Kooner and D.V. Fenby. Aust. J. Chem. 33, 1943 (1980).

9. K.D. Cook, G.G. Jones, and J.W. Taylor. Int. J. Mass. Spectrom. Ion Phys. 35, 273 (1980).

10. J.M. Soler, J.J. Sáenz, N. García, and O. Echt. Chem. Phys. Lett. 109, 71 (1984).

11. (a) M.S. El-Shall, C. Marks, L.W. Sieck, and M. Meot-Ner (mautner). J. Phys. Chem. 96, 2045 (1992); (b) K. Hirao, M. Sano, and Y. Yambe. Chem. Phys. Lett. 87, 181 (1982).

12. W.M. Jackson, D. Xu, R.J. Price, K.L. McNesby, and I.A. McLaren. In Imaging in chemical dynamics, ACS Symposium Series 779. Edited by A. G. Suits and R. E. Continetti. American Chemical Society, Washington, DC, 2001, p. 103.

13. M.J. Frisch, G.W. Trucks, H.B. Schlegel, G.E. Scuseria, M.A. Robb, J.R. Cheeseman, V.G. Zakrzewski, J.A. Montgomery, Jr., R.E. Stratmann, J.C. Burant, S. Dapprich, J.M. Millam, A.D. Daniels, K.N. Kudin, M.C. Strain, O. Farkas, J. Tomasi, V. Barone, M. Cossi, R. Cammi, B. Mennucci, C. Pomelli, C. Adamo, S. Clifford, J.W. Ochterski, G.A. Petersson, P.Y. Ayala, Q. Cui, K. Morokuma, D.K. Malick, A.D. Rabuck,
K. Raghavachari, J.B. Foresman, J. Cioslowski, J.V. Ortiz, A.G. Baboul, B.B. Stefanov, G. Liu, A. Liashenko, P. Piskorz, I. Komaromi, R. Gomperts, R.L. Martin, D.J. Fox, T. Keith, M.A. Al-Laham, C.Y. Peng, A. Nanayakkara, C. Gonzalez, M. Challacombe, P.M.W. Gill, B.G. Johnson, W. Chen, M.W. Wong, J.L. Andres, M. Head-Gordon, E.S. Replogle, and J.A. Pople. GAUSSIAN 98 [computer program]. Revision A.9. Gaussian, Inc. Pittsburgh, PA. 1998.

14. (a) A.D. Becke. J. Chem. Phys. 88, 1053 (1988); (b) C. Lee, W. Wang, and R. Parr. Phys. Rev. B 37, 785 (1988); (c) A. D. Becke. J. Chem. Phys. 98, 5648 (1993).

15. F. Hagmeister, C. Gruenloh, and T. Zwier. J. Phys. Chem. 102, 82 (1998).

16. S.L. Boyd and R.J. Boyd. J. Chem. Theory Comput. 3, 54 (2007).

17. S.F. Boys and F. Bernardi. Mol. Phys. 19, 553 (1970).

18. S.S. Xantheas. Chem. Phys. Lett. 258, 225 (2000).

19. S.S. Xantheas and T.H. Dunning, Jr. J. Chem. Phys. 99, 8774 (1993).

20. F. Huisken, M. Kaloudis, and A. Kulcke. J. Chem. Phys. 104, 17 (1996).

21. L. Gonzáles, O. Mó, and M. Yáńez. J. Chem. Phys. 111, 3855 (1999).

22. Y.J. Hu, H.B. Fu, and E.R. Bernstein. J. Chem. Phys. 125, 154306 (2006).

23. R.W. Larsen, P. Zielke, and M.A. Suhm. J. Chem. Phys. 126, 194307 (2007).

24. Y.J. Hu, H.B. Hu, and E.R. Bernstein. J. Chem. Phys. 125, 154305 (2006)

25. T.D. Fridgen, L. MacAleese, T.B. McMahon, J. Lemaire, and P. Maitre. Phys. Chem. Chem. Phys. 8, 955 (2006).

26. K. Hitao, M. Sano, and S. Yamabe. Chem. Phys. Lett. 87, 181 (1982) 